\title{
Darwin, Mamíferos y Galápagos
}

\author{
Santiago F. Burneo \\ Escuela de Ciencias Biológicas de la Pontificia Universidad Católica del Ecuador. \\ sburneo@puce.edu.ec
}

Entre el 15 de septiembre y el 20 de octubre de 1835, el barco de reconocimiento HMS Beagle navegó por las aguas del Archipiélago de Colón como última parada de su viaje por Sudamérica antes de enrumbar hacia las Islas Sociedad y Nueva Zelanda. El naturalista sin sueldo, que en ese entonces tenía 26 años, resume elegantemente su visita de la siguiente manera:

La historia natural de este archipiélago es notable: Parece un pequeño mundo en sí mismo; la gran mayoría de sus habitantes, tanto vegetales como animales, no se hallan en ningún otro sitio.

Aunque los mejores ejemplos del gran endemismo al que Darwin se refiere se encuentran entre las plantas, reptiles y aves, el estudio de los mamíferos en este rincón del planeta es también muy interesante. Los mamíferos de Galápagos no impresionaron mucho a Darwin quien en su obra Zoología del Beagle solamente incluye descripciones del ratón arrocero de Galápagos. Al revisar su obra El Viaje del Beagle encontramos pocas referencias de mamíferos, en una de las citas se puede encontrar nuevamente a esta especie:

Solo uno de los mamíferos terrestres puede ser considerado indígena, el ratón Mus galapagoensis, confinado a San Cristóbal... pertenece a una división de la familia de los ratones característica de América. En Santiago (James)... hay una rata lo suficientemente distinta del tipo común... de las del Viejo Mundo... dudo mucho que una variedad producto del nuevo y peculiar clima, alimento y suelo al cual está sometida.

Esta cita se refiere al ratón arrocero de Galápagos (actualmente conocido como Aegialomys galapagoensis), especie descrita por G. Waterhouse en 1939 que incluye a dos subespecies: la que observó Darwin habitaba en la Isla San Cristóbal hasta su extinción que resultó por competencia con la rata negra y ratón común introducidos; la segunda subespecie mantiene poblaciones reducidas en la Isla Santa Fe; la parte de la cita que dice " [la] ...división de la familia de roedores de América" es la que conocemos actualmente como familia Cricetidae; la otra especie a la que hace referencia este párrafo, es posiblemente el ratón de Santiago, Nesoryzomys swarthi, descrito por Orr en 1938.

Probablemente una de las citas más interesantes sobre mamíferos en El Viaje del Beagle no se refiere a la presencia de especies notables sino a su notable ausencia: 
... estas islas no son valiosas por el número de especies de reptiles, sino por el de individuos, si recordamos los desgastados senderos hechos por los miles de tortugas gigantes... las muchas tortugas (marinas)... las grandes agrupaciones de Amblyrhinchus terrestres... y los grupos de especies marinas que toman el sol en las rocas de cada isla... debemos admitir que no hay otro cuarto del mundo en el que este orden remplaza los mamíferos herbívoros de manera tan extraordinaria.

Realmente la diversidad de mamíferos en el archipiélago es baja (especialmente si no tomamos en cuenta a los cetáceos) y de mamíferos endémicos es particularmente baja (incluso tomando en cuenta a los cetáceos). La mastofauna de Galápagos incluye dos especies de leones marinos endémicos, dos especies de murciélagos nativos, siete especies de ratones endémicos (cuatro extantes, tres $-\mathrm{y}$ una subespecie - extintos), 26 especies de ballenas y delfines y ocho especies de mamíferos introducidos. Esta diversidad se explica por los patrones y limitaciones de dispersión de este grupo y efectos de disonancia y empobrecimiento relacionados a los modelos de biogeografía de islas.

Los pocos mamíferos terrestres que han podido establecerse en las islas incluyen pocas especies de ratones y murciélagos, carnívoros marinos y cetáceos. Resulta interesante ver que entre los grupos ausentes de las islas, en relación a la diversidad del Ecuador continental, encontramos marsupiales americanos, ungulados, primates, conejos, perezosos, osos hormigueros, armadillos, musarañas y manatíes.
Además de las dos especies de ratones mencionadas anteriormente, en Galápagos sobreviven dos especies más: el ratón de Galápagos de Heller (Nesoryzomys narboroughi) y el ratón de Galápagos de Fernandina ( $N$. fernandinae), ambos endémicos de esta última isla. El ratón de Galápagos de Fernandina se consideraba extinto hasta un trabajo reciente de Dowler y colaboradores quienes redescubrieron algunas poblaciones de ratones de este género.

Tres especies se han extinguido de Galápagos, además de la subespecie de San Cristóbal del ratón arrocero de Galápagos, dos de ellas pertenecientes al género Nesoryzomys, ambas distribuidas originalmente en la isla de Santa Cruz (N. darwini y N. indefessus). Las tres especies de Nesoryzomys no se encuentran taxonómicamente definidas ya que algunos autores recomiendan tratarlas como subespecies de un solo linaje.

La rata gigante de Galápagos, Megaoryzomys curioi, es una especie conocida únicamente por restos óseos y dentales. Estas poblaciones presumiblemente sobrevivieron hasta inicios del siglo XX pero fueron víctimas de depredación y competencia de gatos, perros, chanchos y ratas introducidas.

El grupo de los carnívoros está representado por dos especies de la familia Otariidae. El león marino de Galápagos, una de las tres especies del género Zalophus (las otras dos habitan las costas de Japón y Norteamérica), es endémico del Ecuador, aunque no necesariamente de Galápagos ya que se ha registrado una colonia reciente en la Isla de la Plata. Fueron considerados subespecie de $Z$. californianus hasta que estudios moleculares actuales han validado la especie $Z$. wollebaeki. 
El lobo marino de dos pelos de Galápagos, Arctocephalus galapagoensis, es endémico del archipiélago aunque a mediados del siglo pasado fue considerado subespecie de lobo marino sudamericano, A. australis especie hermana que habita las costas sudamericanas y las Islas Malvinas. Estudios de Repenning y colaboradores demostraron la validez de la especie. El lobo marino sudamericano ha sido registrado ocasionalmente en Galápagos al igual que el león marino del sur (Otaria flavescens).

Pese a la gran capacidad de dispersión de los murciélagos, solamente dos especies de la familia Vespertilionidae han sido capaces de colonizar las islas, y ambas presentan una amplia distribución mundial. El murciélago rojo, Lasiurus blossevillii, se distribuye desde Canadá hasta Argentina e Islas del Caribe; por su lado el murciélago escarchado, $L$. cinereus pese a tener una distribución mundial similar a la especie anterior, en el Ecuador continental no ha sido registrado (todavía...) sino únicamente en las islas Santa Cruz, San Cristóbal, Floreana, Isabela y Santiago.

Los cetáceos están representados por unas 26 especies de las cuales tan solo de tres se puede pensar que sean residentes permanentes, aunque su reproducción en el archipiélago no ha sido confirmada, estos son el delfín nariz de botella, Tursiops truncatus, el delfín piloto de aletas cortas, Globicephala macrorhynchus, y la ballena asesina, Orcinus orca.

La colonización humana ha sido despiadada con las especies nativas de plantas y animales de las islas, principalmente por la introducción de especies domésticas de mamíferos que se han convertido en efi- caces depredadores y competidores de las especies nativas. Posiblemente los más nocivos para las especies de mamíferos nativos sean las ratas negras (Rattus rattus) que ya han sido responsables por la extinción de algunos roedores endémicos. La Estación Científica Charles Darwin (ECCD) ha creado una reserva en los islotes Bainbridge con la finalidad de preservar la especie de la isla Santiago que se encuentra en Peligro Crítico de extinción (lista roja UICN). Programas de erradicación química de ratas de la ECCD han sido exitosos en las islas de Lobos, Mosquera y Pitt.

Asimismo, los gatos ferales han sido erradicados exitosamente de Baltra y del islote Lobos; el Parque Nacional Galápagos (PNG) mantiene proyectos de erradicación de gatos que se complementarán con programas de restauración de hábitats y repatriación de especies nativas. La erradicación combina trampeo, venenos y cacería dirigida con linternas y rifles.

Una de las especies más complicadas de erradicar son los chivos (Capra hircus), pero el trabajo del PNG ha sido arduo y fructífero. Los chivos han sido erradicados de Española, Marchena, Pinta, Santa Fe y Santiago (de la cual se han removido más de 70000 chivos). Los métodos usados incluyen el uso de perros cazadores entrenados con técnicas desarrolladas en Nueva Zelanda que ignoran otras especies y arrean a los chivos hacia los cazadores, cacería con rifles telescópicos desde tierra y helicópteros y el uso de chivos Judas a los que una vez que se les ha colocado un radio transmisor, se los libera en puntos estratégicos de las islas aprovechando la naturaleza gregaria de esta especie para 
encontrar grupos de chivos difíciles de encontrar. Estas técnicas han sido apoyadas por tecnología GPS y de Sistemas de Información Geográfica, sobre todo en la isla Santiago, lo que ha permitido grandes resultados.

Entre lo que queda por hacer en mamíferos de Galápagos, con el apoyo de la comunidad científica y autoridades pertinentes, es documentar detalladamente la historia natural de las especies nativas, evaluar mediante técnicas moleculares sus patrones filogenéticos y desarrollar estrategias de conservación que aseguren la permanencia de los mamíferos en el tiempo.

\section{BIBLIOGRAFIA}

Aydon, C. 2002. Charles Darwin. The naturalist who started a scientific revolution. Avalon Publishing Group, Inc. Londres.

Darwin, C.R. 1839. Voyage of the Beagle: Charles Darwin s Journal of Researches. Browne, J. \& Neve, M., eds, Penguin ( First Edition 1989). Londres.

Darwin, C.R. ed. 1839. Mammalia Part 2 of The zoology of the voyage of HMS Beagle. Smith Elder and Co. Londres.

Dowler, RC, DS Carrol y CW Edwards. 2000. Rediscovery of rodents (Genus Nesoryzomys) considered extinct in the Galapagos Islands. Oryx 34:109-117

Schwartz, D.W. 2008. An Evolution Genius: The extraordinary early life of Darwin Charles. Anthronotes, Museum of Natural History Publication for Educators Vol 29 (2). Washington D.C.

Repenning, CA, RS Peterson y CL Hubbs. 1971. Contributions to the systematics of the southern fur seals, with particular reference to the Juan Fernandez and Guadalupe species. En:WA Burt (Ed.) Antarctic Pinnipedia. Antarctic Research Series 18:1-75 